\title{
Hybrid Optimal Tracking Control by Most Probable Trajectory Approach
}

\author{
Gou Nakura
}

E-mail: gg9925_fiesta@ybb.ne.jp

\begin{abstract}
In this paper, we study optimal tracking control problems for linear continuous-time hybrid systems. We adopt most probable trajectory approach to control system states and mode distributions simultaneously. We consider optimization problems for averaged systems and averaged performance indices throughout mode distributions.

Key Words: Hybrid systems; Optimal tracking control; Most probable trajectory; Mode distributions; Formation
\end{abstract}

\section{Introduction}

On design of hybrid systems composed of multi modes, it is very significant research issue to optimize both system states and modes. It is also very important to consider simultaneous estimation of both system states and inaccessible modes for hybrid systems with unknown modes $[1,3]$. This estimation is called hybrid estimation. By the hybrid estimation we often want to know current mode at each time through information of observation. However there exist cases that we want to know distributions of modes on long run time interval rather than each estimate of the modes themselves at each time to grasp global performance over long time intervals, for example, distributions of active modes in solar systems [2, 6], distributions of active agents on formation or consensus via hybrid systems representation and so on.

In [8], Q. Zhang has presented hybrid filtering algorithm by MPT approach. In the paper, the concerned systems are general hybrid systems which aren't restricted to Markovian jump systems and where added noises aren't restricted to be Gaussian. Throughout the paper, it is assumed that modes of the systems are not directly accessible, and the author has considered optimal estimation problems to find both estimated states of the systems and an optimal candidate of the distributions of the modes over the finite time interval. In the paper most probable trajectory (MPT) approach has adopted to guarantee the optimality of estimation methods. On the approach, given information of observation, he has considered optimal control problems where we seek optimal control by which averaged noises energies are minimized for averaged systems. He has also considered cases that mode transitions follow Markovian jump processes and clarified relationship between estimators based on the Markovian mode transition probabilities and ones based on the limiting probabilities, i.e., he has shown near optimality of the limiting estimators.

G. Nakura has extended the filtering theory by Q. Zhang [8] to smoothing theory by two filters approach $[4,5]$. He has introduced backward filtering diffrential equations, and presented the smoothing algorithm by connecting the solutions of forward filtering equations and those of backward smoothing equations. Also he has presented numerical examples and verified that better estimation performance by smoothing than filtering can be obtained. Note that, on these research, the concerned hybrid systems are transformed to the averaged systems throughout some candidates of mode distributions, and the optimal estimation problems are reduced to some optimal control problems. Therefore we must be able to consider optimal control problems to optimize control inputs and mode distributions simultaneously for general hybrid systems.

In this paper, we study a linear quadratic (LQ) optimal tracking control problems for linear continuoustime hybrid systems over the fixed time interval. The concerned systems are general hybrid systems given below which aren't restricted to Markovian jump systems. It is assumed that modes of the systems are not directly accessible throughout this paper. We consider optimal tracking control problems to find both optimized states of the systems and an optimal candidate of the distributions of the modes for some reference signals over the finite time interval. We adopt most probable trajectory (MPT) approach to guarantee the optimality of tracking control systems. In this paper we modify MPT approach for the state estimation in order to solve the optimal hybrid tracking control problems. On this approach, given information of reference signals, we consider optimal control problems where we seek optimal control by which averaged energies are minimized for averaged systems. Finally we give numerical examples and verify that we can obtain both optimal tracking performance and optimal candidate of mode distributions by the algorithm presented in this paper.

Notations: Throughout this paper the superscript"'" stands for the matrix transposition, $\|\cdot\|$ denotes the Euclidean vector norm and $\|v\|_{R}^{2}$ also denotes the weighted 
norm $v^{\prime} R v$. $O$ denotes the matrix with all zero components.

\section{Problem Formulation}

Let $(\Omega, \mathcal{F}, \mathcal{P})$ be a probability space, where $\Omega$ is the sample space, $\mathcal{F}$ is a $\sigma$-algebra of a subset of $\Omega$ called events and $\mathcal{P}$ is the probability measure on $\mathcal{F}$. On this space, we consider the following system with mode transitions.

$$
\begin{gathered}
\begin{array}{r}
\dot{x}(t)=A(t, \theta(t)) x(t)+B_{2}(t, \theta(t)) u(t, \theta(t)) \\
\\
+B_{3}(t, \theta(t)) r_{c}(t), \\
x(0)=x_{0}, \theta(0)=i_{0} \\
z_{c}(t)=C_{1}(t, \theta(t)) x(t)+D_{12}(t, \theta(t)) u(t) \\
+D_{13}(t, \theta(t)) r_{c}(t)
\end{array}
\end{gathered}
$$

where $x \in \mathbf{R}^{n}$ is the state, $u \in \mathbf{R}^{m}$ is the control input, $z_{c} \in \mathbf{R}^{k_{c}}$ is the controlled output and $r_{c}(\cdot) \in \mathbf{R}^{r_{c}}$ is a known or measurable reference signal. $x_{0}$ is an unknown initial state and it is assumed that a distribution of initial modes $i_{0}$ is given.

We assume that all these matrices are of compatible dimensions. For this system (1), we assume the following conditions:

$$
\text { A1 }: \operatorname{rank} B_{2}(t, \theta(t))=m
$$

$$
\text { A2 : } D_{12}^{\prime}(t, \theta(t)) D_{12}(t, \theta(t)>O
$$

$\theta(t)$ represents a mode sequence of the hybrid system (1). Let $\mathcal{M}=\{1,2, \cdots, M\}$ denote the state space of $\theta(t)$. We define $A(t, 1):=A_{1}(t)$ and so on. In this paper it is assumed that the probability distribution of $\theta(\cdot)$ is unknown, but among a finite number of candidate distributions. Let $r \in \mathcal{N}_{0}=\left\{1,2, \cdots, n_{0}\right\}$, and let $\mathcal{P}=\left\{\phi^{(1)}(\cdot), \cdots, \phi^{\left(n_{0}\right)}(\cdot)\right\}$ denote the set of such candidate distributions on $\mathcal{M}$, i.e., for $r \in \mathcal{N}_{0}$ and $t \in[0, T]$, $\phi^{(r)}(t)=\left(\phi_{1}^{(r)}(t), \cdots, \phi_{M}^{(r)}(t)\right)$ with $\phi_{i}^{(r)}(t) \geq 0$ and $\sum_{i=1}^{M} \phi_{i}^{(r)}(t)=1$.

The optimal tracking control problem on the finite time interval we address in this paper for the system (1) is to find an optimal input and the MPT (most probable trajectory) trajectory of $\theta(t)$ and $x(t), t \in[0, T]$, over the finite horizon $[0, T]$, using the information available on the known part of the information of the reference signal $r_{c}(\cdot)$ for the given distributions of initial mode $i_{0}$ and initial state $x_{0}$.

We define the following performance index for $r \in \mathcal{N}_{0}$ and $t \in[0, T]$ :

$$
\begin{aligned}
J_{0 T}^{(r)}\left(x_{0}, u\right):= & \int_{0}^{T} \sum_{i=1}^{M} \phi_{i}^{(r)}(s) \| C_{1}(s, i) x(s) \\
& +D_{12}(s, i) u(s)+D_{13}(s, i) r_{c}(s) \|^{2} d s \\
& +x^{\prime}(T) Q_{T} x(T)
\end{aligned}
$$

where $Q_{T}>O$ is a symmetric weighting matrix for the terminal state. Thus this performance index means the energies of controlled output and terminal state under some uncertainties averaged by the mode distributions for each $r \in \mathcal{N}_{0}$. We consider the optimization problems to decide $u(\cdot, i)$ and $r \in \mathcal{N}_{0}$ minimizing and $J_{0 T}^{(r)}$ utilizing the known parts of the information of reference signal $\mathcal{R}_{T}=\left\{r_{c}(l) \mid 0 \leq l \leq T\right\}$.

Since the mode $\theta(t)$ at each time is inaccessible, we cannot directly design controllers for the system (1) including the unknown modes. Also, even if the modes are accessible, the computational complexity can exponentially increase if we directly design the controllers for the system (1) including $\theta(t)$ explicitly. Hence we introduce the system averaged through the mode distributions for each $r \in \mathcal{N}_{0}$.

For notational simplicity, we adopt the following notation:

$$
\bar{F}^{(r)}(t)=\sum_{i=1}^{M} \phi_{i}^{(r)}(t) F(t, i)
$$

for a matrix function $F(t, i)$ and $r \in \mathcal{N}_{0}$. Similarly ${\overline{F_{1} F_{2}}}^{(r)}(t)=\sum_{i=1}^{m} \phi_{i}^{(r)}(t) F_{1}(t, i) F_{2}(t, i)$ for matrix functions $F_{1}(t, i)$ and $F_{2}(t, i)$ and so on. Using these notations, we can shift the drift term in the system (1) to $\bar{A}^{(r)}(t)$ as follows:

$$
\dot{x}(t)=\bar{A}^{(r)}(t) x(t)+u(t)+B_{3}(t, \theta(t)) r_{c}(t)
$$

where

$$
\begin{aligned}
& u(t)=u^{(r)}(t) \\
= & \left(A(t, \theta(t))-\bar{A}^{(r)}(t)\right) x(t)+B_{2}(t, \theta(t)) u(t, \theta(t)) .
\end{aligned}
$$

By replacing the control input $u(t, i)$ by $B_{2}^{+}(t, i)\left[\left(\bar{A}^{(r)}(t)-A(t, i)\right) x(t)+u(t)\right]$ where $B_{2}^{+}(t, i)$ is the pseudo inverse matrix of $B_{2}(t, i)$ in the performance index (2), we define

$$
\begin{aligned}
& L^{(r)}(t, x, u) \\
:= & \sum_{i=1}^{M} \phi_{i}^{(r)}(t) \| C_{1}(t, i) x(t)+D_{12}(t, i) B_{2}^{+}(t, i)\left[\left(\bar{A}^{(r)}(t)\right.\right. \\
& \quad-A(t, i)) x(t)+u(t)]+D_{13}(t, i) r_{c}(t) \|^{2} .
\end{aligned}
$$

Then we can define the following performance index:

$$
\begin{aligned}
& J_{t T}^{(r)}(t, x, u(\cdot)) \\
:= & \int_{t}^{T} L^{(r)}(s, x(s), u(s)) d s+x^{\prime}(T) Q_{T} x(T)
\end{aligned}
$$

We consider the optimal control problems to minimize $J_{t T}^{(r)}$ for the given parts of $\mathcal{R}_{T}$. Let $V^{(r)}(t, x)$ and $u^{(r)}(t)$ be the value function of these control problems and the optimal control input for each $r \in \mathcal{N}_{0}$ as follows:

$$
\begin{gathered}
V^{(r)}(t, x):=\inf _{u(\cdot)} J_{t T}^{(r)}(t, x, u(\cdot)) \\
u^{(r)}(t):=\arg \min \left\{J_{t T}^{(r)}(t, x, u(t)): u \in \mathbf{R}^{m}\right\}
\end{gathered}
$$


Let $x^{(r) *}(t)$ be the solution of

$$
\dot{x}(t)=\bar{A}^{(r)}(t) x(t)+u(t)+B_{3}\left(t, \phi^{(r)}(t)\right) r_{c}(t)
$$

under the state history $\left\{x^{(\hat{r}(s) *)}(s): 0 \leq s<t\right\}$ with the given $x_{0}$ and $i_{0}$.

Then define

$$
\hat{r}(t):=\arg \min \left\{V^{(r)}\left(t, x^{(r) *}(t)\right): r \in \mathcal{N}_{0}\right\} .
$$

and

$$
V^{(r)}(t):=V^{(r)}\left(t, x^{(\hat{r}) *}(t)\right)
$$

Then the most probable distribution is $\phi^{(\hat{r}(t))}(\cdot)$. Let $x^{*}(t)=x^{(\hat{r}(t) *)}(t)$ and we have

$$
\begin{aligned}
& V^{(r)}(t) \\
\leq & V^{(r)}(t, x)=J_{t T}^{(r)}\left(t, x, u^{(r)}(t)\right) \leq J_{t T}^{(r)}(t, x, u(t)) .
\end{aligned}
$$

Now we define the following optimal trajectory in the sense of most probable trajectory (MPT).

Definition 2.1 Given the matrices $Q_{T}$, $\left(\hat{r}(t), x^{(\hat{r}) *}(t)\right), t \geq 0$, is called an optimal trajectory (in the MPT sense) if it minimizes $V^{(r)}(t, x)$.

Then we formulate the following optimal hybrid tracking control problems for the performance index (3).

The Optimal Hybrid Tracking Control Problem for Linear Continuous-Time Systems on the Finite Time Interval:

Consider the system (1) and the performance index (3). Find the control input $\left\{u^{*}\right\}$ and the pair $\left(\hat{r}(t), x^{(\hat{r}) *}(t)\right)$, $t \in[0, T]$ minimizing the performance index (3) where the control strategy $u^{*}(t), 0 \leq t \leq T$, is decided based on the state information $\mathcal{X}_{t}=\{x(s) \mid 0 \leq s \leq t\}$ and the causal information of the reference signal $\mathcal{R}_{t}=$ $\left\{r_{c}(l) \mid 0 \leq l \leq t\right\}$ until the time $t$.

\section{$3 \quad$ Hybrid Optimal Tracking Algorithms}

\subsection{Optimal Hybrid Tracking}

The Hamilton-Jacobi-Bellman (HJB) equations associated with the optimal control problem to minimize $J_{t T}^{(r)}$ with regard to $u(\cdot)$ are given as follows:

$$
\begin{aligned}
&-\frac{\partial V^{(r)}(t, x)}{\partial t}=\min _{u}\left\{L^{(r)}(t, x, u, r(t))\right. \\
&+\left.\left(\bar{A}^{(r)}(t) x+u+\bar{B}_{3}^{(r)}(t) r_{c}(t)\right)^{\prime} \frac{\partial V^{(r)}(t, x)}{\partial x}\right\} \\
& V^{(r)}(T, x)=x^{\prime}(T) Q_{T} x(T), r \in \mathcal{N}_{0}
\end{aligned}
$$

Then we obtain the following minimizing $u(\cdot)$.

$$
u^{(r)}(t, x)
$$

$$
\begin{aligned}
=-\bar{D}^{(r)-1}( & t)\left(\left\{{\overline{B_{2}^{+^{\prime}} D_{12}^{\prime} C_{1}}}^{(r)}(t)+\bar{D}^{(r)}(t) \bar{A}^{(r)}(t)\right.\right. \\
& \left.-{\overline{B_{2}^{+^{\prime}} D_{12}^{\prime} D_{12} B_{2}^{+} A}}^{(r)}(t)\right\} x(t) \\
+ & \left.{\overline{B_{2}^{+^{\prime}} D_{12}^{\prime} D_{13}}}^{(t)} r_{c}(t)+\frac{1}{2} \frac{\partial V^{(r)}(t, x)}{\partial x}\right)
\end{aligned}
$$

where $D(t)=B_{2}^{+{ }^{\prime}} D_{12}^{\prime} D_{12} B_{2}^{+}(t)$.

Let

$$
V^{(r)}(t, x)=x^{\prime} X^{(r)}(t) x+b^{(r)}(t)
$$

for some functions $X^{(r)}$ and $b^{(r)}$ with appropriate dimensions.

Then we obtain the following matrix differential equations and scalar equations with terminal conditions:

$$
\begin{aligned}
& \dot{X}^{(r)}(t) \\
& +X^{(r)}(t) \bar{A}^{(r)}(t)+\bar{A}^{(r)^{\prime}}(t) X^{(r)}(t) \\
& -X^{(r)}(t)\left(\bar{D}^{(r)}(t)\right)^{-1} X^{(r)}(t) \\
& -X^{(r)}(t)\left(\bar{D}^{(r)}(t)\right)^{-1}\left({\overline{D_{12}^{\prime} C_{1}}}^{(r)}(t)\right. \\
& \left.+\bar{D}^{(r)} \bar{A}^{(r)}(t)-\overline{D_{12}^{\prime} D_{12}} A^{(r)}(t)\right) \\
& -{\overline{C_{1}^{\prime} D_{12}}}^{(r)}(t)+\bar{A}^{(r)^{\prime}} \bar{D}^{(r)}(t) \\
& \left.-{\overline{A^{\prime} D_{12}^{\prime} D_{12}}}^{(r)}(t)\right)\left(\bar{D}^{(r)}(t)\right)^{-1} X^{(r)}(t), \\
& -{\overline{C_{1}^{\prime} D_{12}}}^{(r)}(t)+\bar{A}^{(r)^{\prime}} \bar{D}^{(r)}(t) \\
& \left.-{\overline{A^{\prime} D_{12}^{\prime} D_{12}}}^{(r)}(t)\right)\left(\bar{D}^{(r)}(t)\right)^{-1} \\
& \times\left({\overline{D_{12}^{\prime} C_{1}}}^{(r)}(t)+\bar{D}^{(r)} \bar{A}^{(r)}(t)-{\overline{D_{12}^{\prime} D_{12}}}^{(r)}(t)\right) \\
& +\bar{A}^{(r)^{\prime}}(t) \bar{D}^{(r)}(t) \bar{A}^{(r)}(t)-\bar{A}^{(r)^{\prime}}(t){\overline{D_{12}^{\prime} D_{12} A}}^{(r)}(t) \\
& -{\overline{A^{\prime} D_{12}^{\prime} D_{12}}}^{(r)}(t) \bar{A}^{(r)}(t)+{\overline{A^{\prime} D_{12}^{\prime} D_{12} A}}^{(r)}(t) \\
& +{\overline{C_{1}^{\prime} C_{1}}}^{(r)}(t)+{\overline{C_{1}^{\prime} D_{12}}}^{(r)}(t) \bar{A}^{(r)}(t) \\
& -{\overline{C_{1}^{\prime} D_{12} A}}^{(r)}(t) \\
& +\bar{A}^{(r)^{\prime}}(t){\overline{D_{12}^{\prime} C_{1}}}^{(r)^{\prime}}(t)-{\overline{A^{\prime} D_{12}^{\prime} C_{1}}}^{(r)^{\prime}}(t)=O \\
& X^{(r)}(T)=Q_{T}
\end{aligned}
$$

$$
\begin{aligned}
& -\dot{b}^{(r)}(t) \\
& \left.\left.=\left[r_{c}^{\prime}(t){\overline{B_{2}^{+^{\prime}} D_{12}^{\prime} D_{13}}}^{(r)^{\prime}}(t)\right] \bar{D}^{(r)-1}(t) \overline{\left[{B_{2}^{+{ }^{\prime}} D_{12}^{\prime} D_{13}}\right.}\right] r_{c}(t)\right] \\
& +r_{c}^{\prime}(t){\overline{D_{13}^{\prime} D_{13}}}^{(r)}(t) r_{c}(t) \\
& \left.-2\left[r_{c}^{\prime}(t){\overline{B_{2}^{+^{\prime}} D_{12}^{\prime} D_{13}}}^{\left(r^{\prime}\right)}(t)\right] D^{(r)-1^{\prime}}(t) \overline{{B_{2}^{+^{\prime}} D_{12}^{\prime} D_{13}}}\right] r_{c}(t), \\
& b(T)=0
\end{aligned}
$$

Now we have the following optimal tracking algorithm, which gives the solution of the Optimal Hybrid Tracking Problem for Linear ContinuousTime Systems.

*** Optimal hybrid tracking control algorithm***

Step 1) Obtain $X^{(r)}(t)$ and $b^{(r)}(t)$ for $r \in \mathcal{N}_{0}$ and $t \in$ $[0, T]$ by solving $(5)$ and $(6)$ with terminal conditions. 
Step 2) Using the above $X^{(r)}(t)$, design the candidate of control inputs

$$
\begin{aligned}
& u^{(r)}(t, x) \\
& =-\bar{D}^{(r)-1}(t)\left(\left\{{\overline{B_{2}^{+\prime} D_{12}^{\prime} C_{1}}}^{(r)}(t)+\bar{D}^{(r)}(t) \bar{A}^{(r)}(t)\right.\right. \\
& \left.-{\overline{B_{2}^{+{ }^{\prime}} D_{12}^{\prime} D_{12} B_{2}^{+} A}}^{(r)}(t)\right\} x(t) \\
& \left.+\overline{B_{2}^{+^{\prime}} D_{12}^{\prime} D_{13}}(t) r_{c}(t)+X^{(r)}(t) x(t)\right)
\end{aligned}
$$

and obtain the solution $x^{(r) *}(t)$ of the differential equation

$$
\begin{aligned}
\dot{x}(t)= & \bar{A}^{(r)}(t) x(t)+u(t)+B_{3}\left(t, \phi^{(r)}(t)\right) r_{c}(t), \\
& x(t-\delta t)=x_{0}
\end{aligned}
$$

for small $\delta t>0$.

Step 3) Choose $\hat{r}(t)$ that minimizes $V^{(r)}(t, x)$.

Step 4) For $t+\delta t$, repeat Step 2) and Step 3), and obtain $\hat{r}, u^{(\hat{r}) *}$ and $x^{(\hat{r}) *}$.

Step 5) Repeat from Step 2) to Step 4) until $T$.

Then the most probable distribution is $\phi^{(\hat{r}(t))}(\cdot)$ and the optimal filter is given by

$$
\left(\hat{r}(t), x^{*}(t)\right)=\left(\hat{r}(t), x^{(\hat{r}) *}(t)\right) .
$$

\section{Numerical Examples}

In this section, we study numerical examples to demonstrate the effectiveness of the presented design algorithm.

We consider the following two mode systems and assume that the system parameters are as follows:

$$
\begin{gathered}
\dot{x}(t)=A(t, \theta(t)) x(t)+B_{2}(t) u(t, \theta(t)) \\
+B_{3}(t) r_{c}(t), \\
x(0)=x_{0}, \theta(0)=i_{0} \\
z_{c}(t)=C_{1}(t) x(t)+D_{12}(t) u(t)+D_{13}(t) r_{c}(t)
\end{gathered}
$$

where

$$
\begin{gathered}
\text { Mode 1: } \\
A_{1}=\left[\begin{array}{cc}
0 & 1 \\
-1 & -0.4
\end{array}\right], \quad A_{2}=\left[\begin{array}{cc}
0.5 & 1 \\
0.8 & -0.2
\end{array}\right], \\
B_{2}=\left[\begin{array}{l}
1 \\
1
\end{array}\right], B_{3}=\left[\begin{array}{l}
1 \\
0
\end{array}\right], \\
C_{1}=\left[\begin{array}{cc}
-0.5 & 0.1 \\
0 & 0
\end{array}\right], D_{12}=\left[\begin{array}{l}
1 \\
1
\end{array}\right], D_{13}=\left[\begin{array}{c}
-1 \\
0
\end{array}\right] .
\end{gathered}
$$

We set $\hat{x}_{0}=\operatorname{col}(-0.1,0)$ and the distribution of the initial mode $i_{0}$ as $(1 / 2,1 / 2)$. The candidates of mode distributions are given as follows:

Case I:

$$
\phi^{(1)}=\left(\frac{2}{5}, \frac{3}{5}\right), \phi^{(2)}=\left(\frac{1}{10}, \frac{9}{10}\right), \phi^{(3)}=\left(\frac{7}{10}, \frac{3}{10}\right)
$$

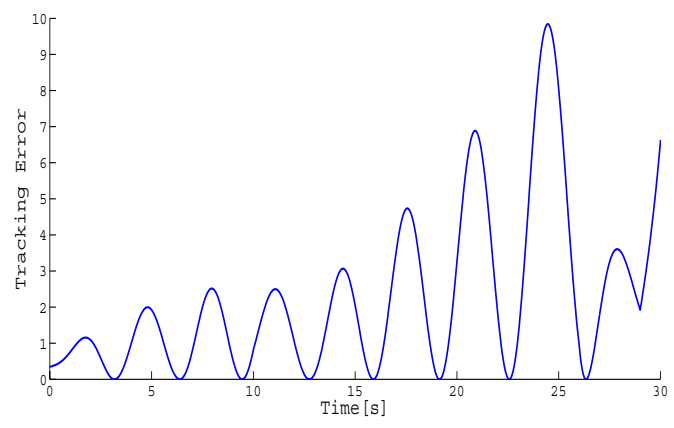

Fig. 1: The value of tracking error for $r_{c}(t)=\sin (t)$ and Case I

$$
\begin{aligned}
& \text { Case II: } \\
& \phi^{(1)}=\left(\frac{2}{5}, \frac{3}{5}\right), \phi^{(2)}=\left(\frac{3}{5}, \frac{2}{5}\right), \phi^{(3)}=\left(\frac{7}{10}, \frac{3}{10}\right)
\end{aligned}
$$

The paths of $\theta(t)$ are generated randomly, and the performances are compared under the same circumstance, that is, the same set of the paths so that the performances can be easily compared.

We verify the effectiveness of the presented hybrid tracking control algorithm for reference signals $r_{c}(t)=$ $\sin (t)$ and $r_{c}(t)=\sin (\pi t / 30)$. In order to carry out these algorithms we solve the backward pair of the differential equations (5) and (6) with the terminal conditions for the given $r_{c}(t)$ and each candidate $r=1,2,3$ of given distributions, and obtain the pair $\left(\hat{r}(t), x^{*}(t)\right)$ minimizing $V_{f}^{(r)}(t)$ for $t \in[0, T]$.

Fig. 1 and Fig. 3 show the values of $\| C_{1} x(t)+$ $D_{13} r_{c}(t) \|^{2}$ for $r_{c}(t)=\sin (t)$ in the cases I and II respectively. Fig. 2 and Fig. 4 show the optimal mode distributions for $r_{c}(t)=\sin (t)$ in the cases I and II respectively. By these figures it is shown that we can obtain better tracking performance in the Case II than the Case I for $r_{c}(t)=\sin (t)$. Fig. 5 and Fig. 7 show the values of $\left\|C_{1} x(t)+D_{13} r_{c}(t)\right\|^{2}$ for $r_{c}(t)=\sin (\pi t / 30)$ in the cases I and II respectively. Fig. 6 and Fig. 8 show the optimal mode distributions for $r_{c}(t)=\sin (\pi t / 30)$ in the cases I and II respectively. By these figures it is shown that we obtail almost the same tracking performances in the Case I and II for $r_{c}(t)=\sin (\pi t / 30)$. These results show that the optimized tracking performances and mode distributions depend on the types of the reference signals numerically while we may prepare the candidates of mode distribution, the performance index and the reference signals independently.

\section{Concluding Remarks}

In this paper we have studied the LQ optimal tracking control problems for linear continuous-time hybrid systems over the fixed time interval. The systems aren't restricted to the Markovian jump systems. We have 


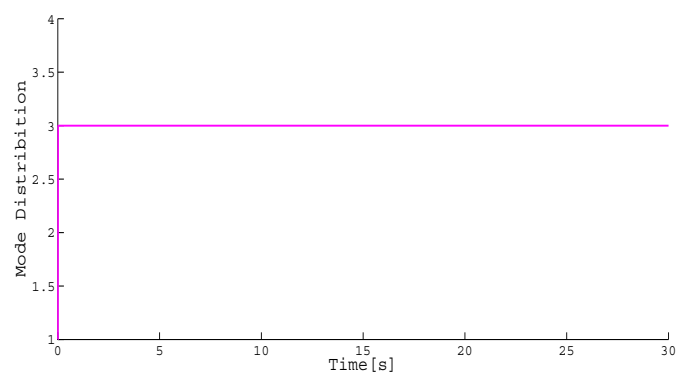

Fig. 2: The optimized mode distribution for $r_{c}(t)=$ $\sin (t)$ and Case I

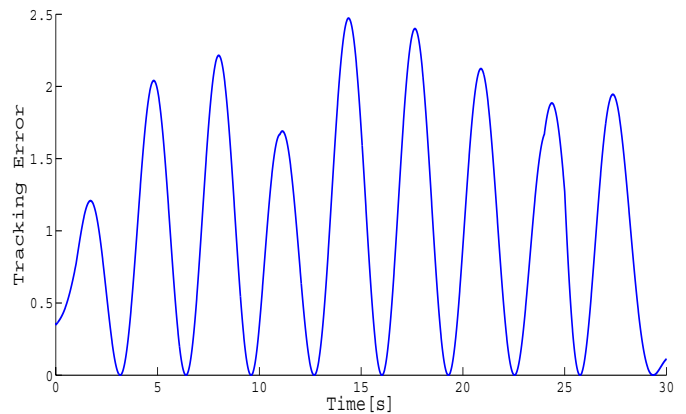

Fig. 3: The value of tracking error for $r_{c}(t)=\sin (t)$ and Case II

adopted the modified MPT approach to solve the optimal hybrid tracking control problems. The optimal tracking approach adopted in this paper guarantees the optimality of tracking performance in the meaning of MPT.

In this paper we have considered the problems that both the system state and modes are optimized. However we have considered the problems that the distributions of the modes over the fixed time interval not the modes themselves are optimized to grasp the global behavior of the hybrid systems over the long time intervals. In order to optimize both the system state and distributions of the modes for the given reference signals we have introduced the averaged performance index with respect to the candidates of the mode distributions for the averaged systems. For this performance index we have formulated the optimal tracking control problem for the information of the reference signals. We have derived the backward Riccati type differential equations, which give the necessary conditions for the solvability of the optimal control problems. Then we have presented the optimal hybrid tracking control algorithm. Finally we have studied the numerical examples to compare the tracking performances.

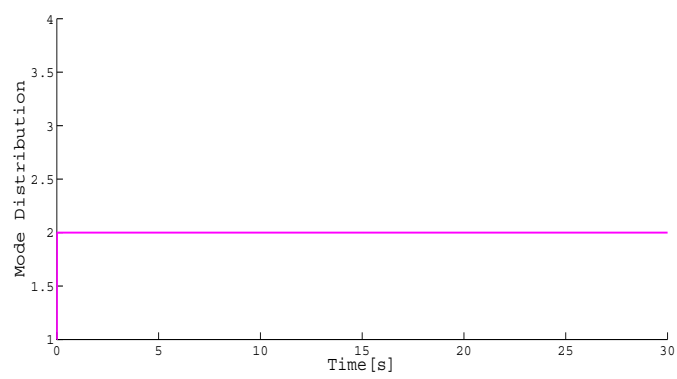

Fig. 4: The optimized mode distribution for $r_{c}(t)=$ $\sin (t)$ and Case II

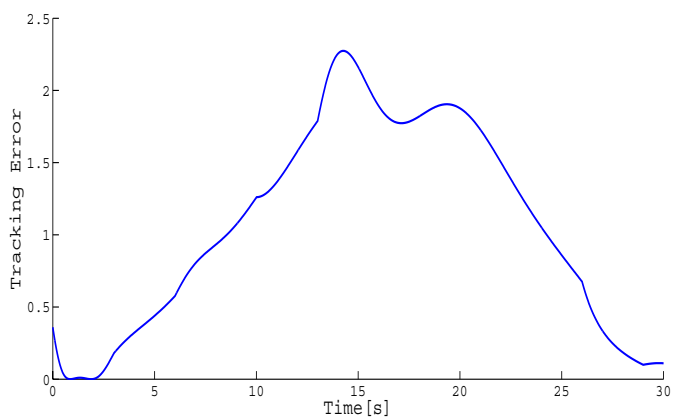

Fig. 5: The value of tracking error for $r_{c}(t)=\sin (\pi t / 30)$ and Case I

\section{References}

[1] O. L. V. Costa: Linear Minimum Mean Square Error Estimation for Discrete-Time Markovian Jump Linear Systems, IEEE Trans. Automat. Contr., Vol. 39, No. 8, pp.1685-1689, 1994.

[2] O. L. V. Costa, M. D. Fragoso and R. P. Marques: Discrete-Time Markov Jump Linear Systems, Springer, London, 2005.

[3] M. D. Fragoso, O. L. V. Costa, J. Baczynski and N. Rocha: Optimal linear mean square filter for continuous-time jump linear systems, IEEE Trans. Automat. Contr., Vol. 50, No. 9, pp.1364-1369, 2005.

[4] G. Nakura: An Approach to Noncausal Hybrid Estimation for Linear Continuous-Time Systems with Non-Gaussian Noises, Proceedings of SICE Annual Conference 2011, Tokyo, Japan, WeC1102, pp. 979-984, (CD-ROM), 2011.

[5] G. Nakura: An Approach to Hybrid Smoothing for Linear Continuous-Time Systems with NonGaussian Noises, Proceedings of the 43rd ISCIE International Symposium on Stochastic Systems 


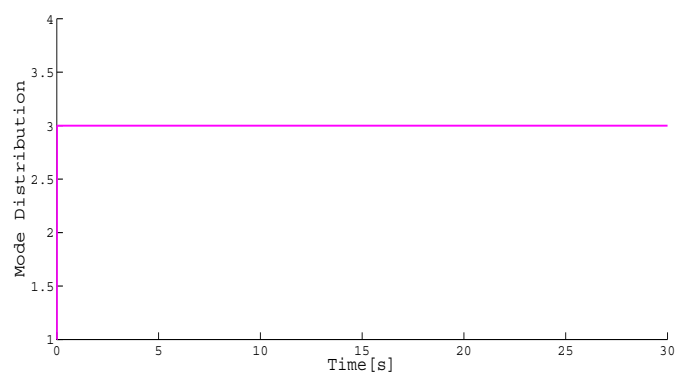

Fig. 6: The optimized mode distribution for $r_{c}(t)=$ $\sin (\pi t / 30)$ and Case I

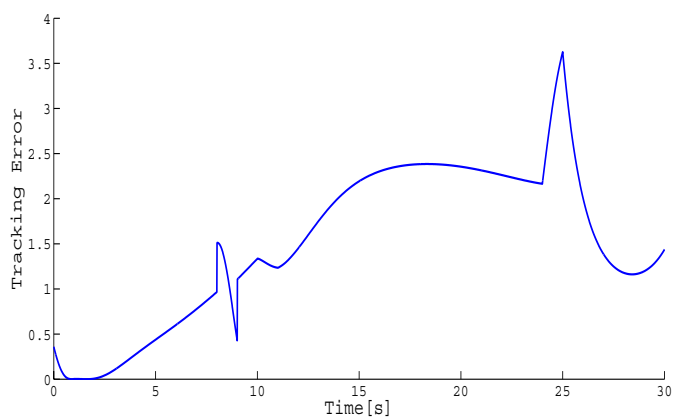

Fig. 7: The value of tracking error for $r_{c}(t)=\sin (\pi t / 30)$ and Case II

Theory and Its Applications (SSS11), Shiga, Japan, pp. 63-72, FB2-2, (CD-ROM), 2011.

[6] D. D. Sworder and R. O. Rogers: An LQG Solution to a Control Problem with Solar Thermal Receiver, IEEE Trans. Automat. Contr., Vol. 28, pp.971-978, 1983.

[7] Q. Zhang: 'Optimal Filtering of Discrete-Time Hybrid Systems', J. Optim. Theory Appl., Vol. 100, No. 1, pp. 123-144, 1999.

[8] Q. Zhang: Hybrid Filtering for Linear Systems with Non-Gaussian Disturbances, IEEE Trans. Automat. Contr., Vol. 45, pp. 50-61, 2000.

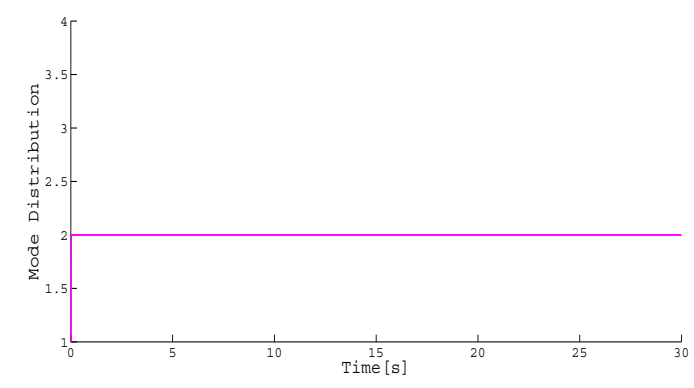

Fig. 8: The optimized mode distribution for $r_{c}(t)=$ $\sin (\pi t / 30)$ and Case II 